\title{
Search for Natural Alkaloids as SARS-CoV-2 Proteas and RdRp Inhibitors: A Docking-Based Study
}

\author{
Abhrajit Bag ${ }^{1}$ and Arijit Bag ${ }^{2 *}$ \\ ${ }^{1}$ Malancha Shishu Shiksha Niketan, Bara Jaguli, West Bengal, India \\ ${ }^{2}$ Department of Applied Science, Maulana Abul Kalam Azad University of \\ Technology, West Bengal, India \\ *Corresponding Author: Arijit Bag, Department of Applied Science, Maulana Abul \\ Kalam Azad University of Technology, West Bengal, India.
}

Received: August 24, 2020

Published: October 28, 2020

(C) All rights are reserved by Abhrajit Bag and

\section{Arijit Bag.}

\begin{abstract}
To find an immediately applicable drug for the treatment of COVID-19 positive patients, a plausible life cycle of this virus is proposed from the analysis of a few case reports. Based on this proposal and symptomatic similarities, a few common drug-molecules are tested as a protease inhibitor and RNA dependent RNA polymerase (RdRp) inhibitor of SARS-CoV-2 virus using COVID-19 Docking Server. It is observed that anisotine and vasicoline of Justicia adhatoda and Pemirolast are very good inhibitors. As all these three compounds are market available drugs, an immediate clinical trial is plausible which may lead to the golden success against the present pandemic. We prescribed different drugs for different purposes and stages of viral infection.
\end{abstract}

Keywords: COVID-19; Structure of SARS-CoV-2; Infection Mechanism of COVID-19; Model Drugs for COVID-19; Docking Study

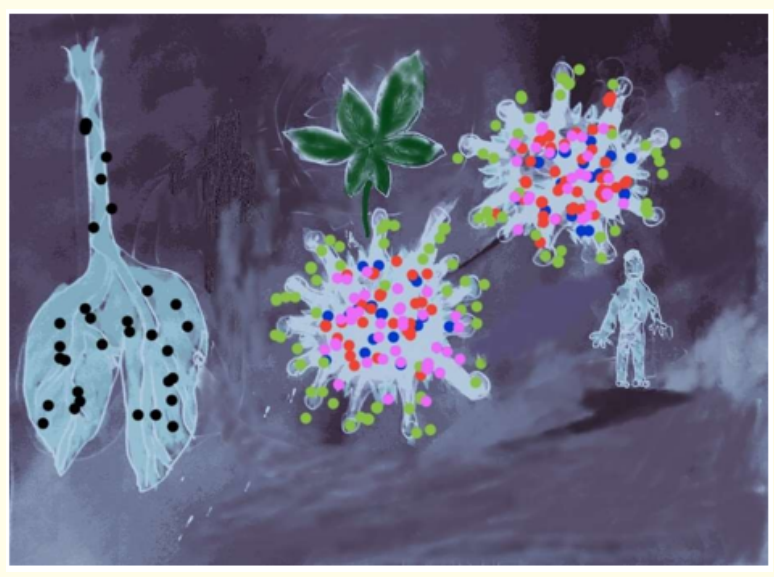

Figure

\section{Introduction}

In the present stage of a worldwide outbreak caused by $\mathrm{CO}$ VID-19 virus [1-5] immediate strong remedy is required, though, there is limited knowledge about this virus and data regarding its effects in the host body and the role of different proteins and enzymes during its life cycle. At present we are also facing a stout challenge and in a race with its propagation to the quest for its recuperation. To infringe on this hurdle, we have to be precise about our planning and placid with the comfort of limitations. We divided our assignment into different steps as follows to knit our roadmap towards fruition:

- Step-1: Review of the structure, infection mechanism and probable life cycle of the virus.

- $\quad$ Step-2: Selection of probable drugs within the available drug bank or easily available source.

- Step-3: Finding of advanced way to prevent transmission.

- $\quad$ Step-4: Docking study with a small set of selected compounds to test their efficacy against COVID-19.

Structure, infection mechanism, and probable life cycle of SARS-CoV-2

According to the world health organization (WHO), presently more than 200 compounds are registered for clinical trials as COV- 
ID-19 drugs. But, to date, the search for a potential drug against this virus is on and more efforts are demanded. In the present research work, we model/select drugs/drug-like compounds for the docking test according to different stages of viral load in the affected body and different stages of the life cycle of the virus in the host body. Of course, only a few are known to us about this virus infection mechanism and the role of different proteins and enzymes during its complete life cycle inside its host body. We build our planning based on the limited knowledge available so far from different case studies and scientific reports. We analyze the structure, source and mode of infection, place of infection and the symptomatic effects observed in the affected person. As the detailed mechanism of infection by the COVID-19 virus is not available, we followed the common way of propagation of a virus inside a living organism. Report on different case studies [6-8] is used to make potential assumptions in favour of our study.

COVID-19 is a retrovirus with positive sign RNA [9] and the latest member of the coronavirus family [10]. Its RNA sequencing has been done and reported [11,12]. It has very close homology similarity with bat coronavirus (96\%) and SARS coronavirus (87\%). It is also known that this virus is transmitted from an affected body or infected body to another body through small droplets due to sneezing and coughing of the ill person or an asymptomatic carrier [13]. The virus enters into a new host body through his respiratory system. Fatality occurs due to the multi-organ failure; particularly, heart attack and respiratory problems. Now, the question is, how does this virus affect other organs except the respiratory system? We are asking this question, because, the progress of infection in a body is very important to understand the mechanistic pathway of the virus life cycle. A common way of propagation of viral infection is migration through blood circulation. But it may not be the case for COVID-19. A recent study by Chen., et al. [14] reported that pregnant women who were COVID-19 positive gave birth of babies with COVID-19 negative. Not only that, cord blood, amniotic fluid, breast milk, and neonatal throat swab are also reported as negative COVID-19. Though all pregnant women under this study recovered from COVID-19, we may conclude that this virus does not transport through blood and not migrated to other organs that are not directly connected to our respiratory system. In favour of our conclusion, there is another case study reported by Bai., et al. where it was observed that the virus is transmitted from a patient who was not severely affected by this virus. Thus, the transmission of the virus occurs through our respiration, sneezing, and coughing.
So, our question remains. We need more evidence to find out how other organs failure occurs. A recent study by liu wenzhong and Li hualan [15] reported that 'orf1ab, ORF10, and ORF3a proteins could coordinately attack heme to dissociate the iron to form the porphyrin'. This observation suggests that the virus fused in the alveolar cells and get replicated there. New matured viruses are released from these cells and ejected from the lungs through our respiration. This is the complete life cycle of this virus. In this way, alveolar cells are destroyed and at the same time hemoglobin is disintegrated. The presence of patches in the lungs observed in the CT scan is proof in favor of this claim [14]. Both of these processes create respiratory failure. These also reduce the oxygen absorption from lung and carbon-di-oxide removal from body cells. Lack of oxygen increases the weakness of the patients and the increase of carbon- di-oxide in cells increases the toxicity which eventually creates other organ failures like a heart attack. This conclusion is very helpful to plan our treatment against COVID-19. We have to focus our study on the respiratory system only.

\section{Methodology \\ Compound selection}

Selection of probable drugs within the available drug bank or easily available source Considering the present situation, we tried to select our targeted drugs within a drug bank or compounds which are available from very common sources. Finding out a completely new compound as a potential drug would not be effective as that would take time to be properly characterized and synthesized but the demand is very high and the urgency. Presently, hydroxychloroquine (MD1) is used for the treatment of COVID-19 affected patients in India which is approved by the Indian Council of Medical Research (ICMR). But this selection is not scientifically tested. This is approved based on common knowledge about this compound. This fact shows the urgency of COVID-19 drug discovery, worldwide. Thus, we chose this compound for our study. We have also chosen 4-Methyl-2-propylquinoline (MD2) for our study as this compound is of a similar class of MD1 and used as an antituberculosis drug. Our third compound is pemirolast (MD3). We chose this compound because a recent super- computer-based docking study over 9127 ligands from the SWEETLEAD database showed the highest drug potency of this compound to inhibit the attachment of COVID-19 virus by a docking study targeted to the interface of the spike protein of the virus and human ACE2 receptor [16]. The rank score of this compound is reported as - $7.4 \mathrm{kcal} /$ 
mol. This compoundis also used as a potential drug for asthma. It is known that the incubation period of this virus is very high which is on an average of 14 days. Thus, it could be considered that the fusion period of this virus is large and a drug like MD3 could be used to stop its fusion. To do that, this drug could be used through nebulization for suspected patients. We test the drug potency of this compound as the protease and RdRp inhibitor. We chose two more compounds vasicoline (MD4) and anisotine (MD5) which are alkaloids obtained from Justicia adhatoda leaves. Both these compounds are also used as a drug for diseases related to respiratory problems. Justicia adhatoda which is commonly known as Basak is available all over India and frequently used for home treatment of cold and cough. Thus, there is no risk at all of taking these medicines. We may also use the fresh juice obtained from Basak leaves where it is available as a precaution. Of course, in Justicia adhatoda leaves there are few more alkaloids we chose only these two randomly as we had no computational facilities. We used COVID-19 Docking Server, which is a web server, for our computation. Thus, we are restricted and due to the present crisis, we could not wait for more tests as we have obtained at least three good drug- like compounds for the COVID-19 virus. We also performed similar tests for ethambutol (MD6) which is a popular drug for tuberculosis. We chose this drug due to its symptomatic similarities with COVID-19. All these compounds are easily market available drugs. Thus, their properties are well known and hence could be directly promoted for clinical trials with minimum risks.

\section{Prevention of transmission}

As discussed earlier that transmission occurs through our respiratory system, we could prevent it by using a proper inhaler. Pemirolast could be used for this purpose as it showed potency to stop the fusion process of the virus. It could be used for those who are suspected of virus contamination. But, how the spread of the virus from a severely affected patient could be stopped is not sure from this study. Though we may take a test of it using the drug prescribed here for the other purpose. Prevention of transmission from different mediums is well discussed in the literature. Thus, we are not going for that.

\section{Docking study to test efficacy against COVID-19}

To test the efficacy of our selected compounds we perform molecular docking studies. All dockings are performed with COVID-19 Docking Server [17] provide by R. Kong., et al. In this server different proteins of COVID-19 virus are available for immediate docking with small molecules and peptides. We used all pre-decided param- eters for docking. We focused only on protease inhibition and RdRp inhibition (RdRp) for our study. Docking results are presented in table 1.

\begin{tabular}{|l|c|c|}
\hline Compounds & $\begin{array}{c}\text { Binding } \\
\text { energy with } \\
\text { protease } \\
\text { (kcal/mol) }\end{array}$ & $\begin{array}{c}\text { Binding energy } \\
\text { with replicase } \\
\text { (RdRp) (kcal/ } \\
\text { mol) }\end{array}$ \\
\hline $\begin{array}{l}\text { Hydroxychloroquine } \\
\text { (MD1) }\end{array}$ & -6.3 & -7.1 \\
\hline $\begin{array}{l}\text { 4-Methyl-2-propylquino- } \\
\text { line (MD2) }\end{array}$ & -5.8 & -7.6 \\
\hline Pemirolast (MD3) & -6.9 & -8.9 \\
\hline Vasicoline (MD4) & -7.0 & -8.5 \\
\hline Anisotine (MD5) & -7.8 & -9.2 \\
\hline Ethambutol (MD6) & -5.1 & -6.1 \\
\hline
\end{tabular}

Table 1: Docking results of modeled compounds with SARS-CoV-2 protease and RdRp.

\section{Results}

From the docking results presented in table 1, we observed that MD5 has the highest activity of inhibition for both protease and $\mathrm{RdRp}$. The binding energies are -7.8 and $-9.2 \mathrm{kcal} / \mathrm{mol}$ respectively. The second highest protease inhibition activity is observed for MD4 which is $-7.0 \mathrm{kcal} / \mathrm{mol}$ which is not excellent but acceptable. But, RdRp inhibition activity of this compound is very good, $-8.5 \mathrm{kcal} /$ mol. Thus, these two compounds could be very good drugs for the treatment of COVID-19 patients. These two compounds are highlighted with green color in table 1 . There is another compound that has the second-highest activity against RdRp after MD5. It is MD3 which has reported for a good activity to inhibit virus fusion. The RdRp inhibition activity of MD3 is $-8.9 \mathrm{kcal} / \mathrm{mol}$. It is also highlighted with green color in the table. From their binding energies, it is expected that all three compounds could show activity at the nanomolar level. Here it should be noted that both protease and RdRp protein binding energy values of compounds MD3, MD4 and MD5 are better than MD1 (-6.3 and $-7.1 \mathrm{kcal} / \mathrm{mol})$ which is presently used for COVID-19 affected patients' treatment in the absence of any full proved drug. Our study suggested that compounds MD3, MD4, and MD5 are better compared to MD1. As MD1 is reported as good for this treatment, our proposed compounds MD3, MD4 and MD5 will produce better results. Protease inhibition activity of MD2 is very poor, the rank score is only $-5.8 \mathrm{kcal} / \mathrm{mol}$. So as MD6, $-5.1 \mathrm{kcal} / \mathrm{mol}$. But, RdRp activity of MD2 is quite good and accept- 
able, value if $-7.6 \mathrm{kcal} / \mathrm{mol}$ which is better than MD1. Similar is not true for MD6. Its RdRp binding energy is only $-6.1 \mathrm{kcal} / \mathrm{mol}$, minimum among all modelled compounds.

\section{Discussions}

The results of our study proclaim that MD3, MD4, and MD5 compounds could be used as a drug for COVID-19 treatment instead of MD1 or MD2. As all these compounds are market available drugs their toxicity, LogP, cell uptake, EC50, etc. values are known. We need not any study regarding these values. Their effects on the human body are also known. Thus, we should directly go for the clinical trial with these compounds as there is practically no time in our hands as the total death due to the COVID-19 virus for the last 24 hours (1.22 am IST, 27 $7^{\text {th }}$ March 2020) is 2345 and new cases of COVID-19 positive is 51781. We may prescribe remedy from COVID-19 from our present study as follows:

- For precaution: Justicia adhatoda leaves may be boiled with freshwater and its vapor may be inheld while the extract may be drunk after proper filtration as a hot health drink.

- $\quad$ For suspected: MD3 may be used through an inhaler. MD4 and MD5 may also be effective for this purpose but the present study does not confirm that. The proper test is required before the confirmed prescription.

- For active cases at the mild condition: MD5 along MD3 or MD4 may be used orally for a clinical test with variable doges depending upon the condition of the patient.

- For active cases at the critical condition: MD5 may be used orally along with MD3 through an inhaler. Here it is important to say that these three suggested compounds are supposed to work through the inhibition of viral protease and RdRp. But, for a critical patient, a lot of damage to the alveolar cell has been done. Thus, the only stopping of viral replication would not be helpful to cure. We need supportive and symptomatic treatment side by side. As it is discussed earlier that this virus destroys hemoglobin which also causes oxygen deficiency of the patient and responsible for multiorgan failure and heart attack, blood (RBC) transplantation is essential.

\section{Conclusion}

Fight against COVID-19 is the primary concern of all around the globe at this instant. Most of the countries are in a lockdown situation. In this respect, our study is highly relevant. The results of our study are very impressive and encouraging. Though the drug response is not estimated, from the inhibition constant of MD5 we may conclude that RdRp inhibition activity of this compound would be in the nanomolar range. The stage-wise drug selections prescribed in the discussion section may be very helpful to stop this pandemic. As these compounds are market available drugs, application approval from the proper authority would be immediately implemented without any real-time delay and difficulties. Thus, these compounds demand clinical trials at the earliest convenience.

\section{Acknowledgments}

Both the authors are thankful to COVID-19 Docking Server providers for giving the opportunity of docking. Arijit Bag likes to thank Dona Pal for raising inspiration towards this work.

\section{Bibliography}

1. World Health Organization. "Coronavirus disease 2019 (COVID-19): situation report" (2020): 51.

2. Liu Y., et al. "The reproductive number of COVID- 19 is higher compared to SARS coronavirus". Journal of Travel Medicine (2020): taaa21.

3. Pan F., et al. "Time course of lung changes on chest CT during recovery from 2019 novel coronavirus (COVID-19) pneumonia”. Radiology (2020): 200370.

4. Wu Z and McGoogan JM. "Characteristics of and important lessons from the coronavirus disease 2019 (COVID-19) outbreak in China: summary of a report of 72314 cases from the Chinese Center for Disease Control and Prevention". The Journal of the American Medical Association (2020).

5. Fang Y., et al. "Sensitivity of chest CT for COVID-19: comparison to RT-PCR”. Radiology (2020): 200432.

6. Ai T., et al. "Correlation of chest CT and RT-PCR testing in coronavirus disease 2019 (COVID-19) in China: a report of 1014 cases". Radiology (2020): 200642.

7. Shi H., et al. "Radiological findings from 81 patients with COVID-19 pneumonia in Wuhan, China: a descriptive study". The Lancet Infectious Diseases (2020).

8. Lan L., et al. "Positive RT-PCR test results in patients recovered from COVID-19". The Journal of the American Medical Association (2020).

9. Yan R., et al. "Structural basis for the recognition of the SARSCoV-2 by full-length human ACE2". Science (2020). 
10. Guarner J. "Three Emerging Coronaviruses in Two Decades: The Story of SARS, MERS, and Now COVID-19 (2020).

11. Kim JM., et al. "Identification of Coronavirus Isolated from a Patient in Korea with COVID-19". Osong Public Health and Research Perspectives 11.1 (2020): 3.

12. He Q., et al. "Single Cell RNA Sequencing Analysis of Human Kidney Reveals the Presence of ACE2 Receptor: A Potential Pathway of COVID- 19 Infection (2020).

13. Bai Y., et al. "Presumed asymptomatic carrier transmission of COVID-19". The Journal of the American Medical Association (2020).

14. Chen H., et al. "Clinical characteristics and intrauterine vertical transmission potential of COVID-19 infection in nine pregnant women: a retrospective review of medical records". The Lancet 395.10226 (2020): 809-815.

15. Liu W and Li H. "COVID-19 Disease: ORF8 and Surface Glycoprotein Inhibit Heme Metabolism by Binding to Porphyrin". Chem Rxiv (2020).

16. Smith M and Smith JC. "Repurposing Therapeutics for COVID-19: Supercomputer-Based Docking to the SARS-CoV-2 Viral Spike Protein and Viral Spike Protein-Human ACE2 Interface". Chem Rxiv (2020).

17. Kong R., et al. "COVID-19 Docking Server: An interactive server for docking small molecules, peptides and antibodies against potential targets of COVID-19 (2003).

\section{Assets from publication with us}

- Prompt Acknowledgement after receiving the article

- Thorough Double blinded peer review

- Rapid Publication

- Issue of Publication Certificate

- High visibility of your Published work

Website: www.actascientific.com/

Submit Article: www.actascientific.com/submission.php

Email us: editor@actascientific.com

Contact us: +919182824667

Citation: Abhrajit Bag and Arijit Bag. "Search for Natural Alkaloids as SARS-CoV-2 Proteas and RdRp Inhibitors: A Docking-Based Study". Acta Scientific Pharmaceutical Sciences 4.11 (2020): 77-81. 